\title{
Spontaneous elbow hemarthrosis identified by point-of-care ultrasound
}

eISSN: 2383-4625

\author{
David C. Mackenzie, Scott McCorvey \\ Department of Emergency Medicine, Maine Medical Center, Portland, ME, USA
}

Traumatic or spontaneous hemarthroses are an important cause of joint effusions, and can complicate innate or acquired coagulopathies. The elbow is an unusual location for a spontaneous hemarthrosis; we describe a previously unreported case of warfarin-induced spontaneous elbow hemarthrosis, diagnosed by point-of-care ultrasound. On the basis of clinical and ultrasound findings arthrocentesis was deferred, and the patient was successfully treated with warfarin reversal and conservative care. Physical examination is unreliable for the detection of a joint effusion, and misdiagnosis and can lead to unnecessary investigation or resource use. Point-of-care ultrasound allows accurate, prompt, direct visualization of a joint effusion, and non-invasive confirmation of a hemarthrosis. Ultrasound can facilitate accurate diagnosis and characterization of joint effusions to improve the care of patients with coagulopathy.

Keywords Ultrasonography; Joints; Hemarthrosis; Warfarin

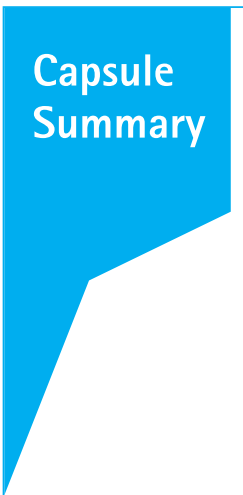

What is already known

Spontaneous hemarthrosis can be a complication of anticoagulant therapy, but typically affects weight-bearing joints in the lower extremity. Physical examination for the detection of hemarthrosis is unreliable.

What is new in the current study

We describe a previously unreported case of warfarin-related spontaneous elbow hemarthrosis. The hemarthrosis was identified with point-of-care ultrasound.
Received: 20 February 2016

Revised: 20 April 2016

Accepted: 10 May 2016

Correspondence to: David C. Mackenzie Department of Emergency Medicine, Maine Medical Center, 22 Bramhall Street, Portland, ME 04102, USA E-mail: DMackenzie@mmc.org

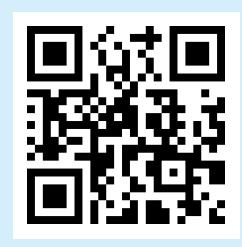

How to cite this article:

Mackenzie DC, McCorvey S. Spontaneous elbow hemarthrosis identified by point-ofcare ultrasound. Clin Exp Emerg Med 2017;4(1):60-63.

This is an Open Access article distributed under the terms of the Creative Commons Attribution Non-Commercial License (http:// creativecommons.org/licenses/by-nc/4.0/). 


\section{INTRODUCTION}

Musculoskeletal complaints comprise a large percentage of emergency department visits across all age groups. ${ }^{1}$ Point-of-care ultrasound can facilitate the timely diagnosis and treatment of patients with musculoskeletal complaints, and may be superior to clinical examination or radiographs for detecting joint effusions. ${ }^{2-4}$ Increasing evidence supports the use of ultrasound in the emergency department for the diagnosis and treatment of fractures, tendon injuries, joint effusions, and soft tissue infections..$^{5-7} \mathrm{We}$ report a novel case of a man with a spontaneous elbow hemarthrosis in the setting of a warfarin-induced coagulopathy. The hemarthrosis was diagnosed using point-of-care ultrasound. This case illustrates how point-of-care ultrasound may be used to characterize a hemarthrosis and inform a clinician's decision to perform or defer arthrocentesis and to guide treatment.

\section{CASE REPORT}

A 79-year-old male presented to the emergency department with acute left elbow pain. His past medical history was significant for diabetes mellitus, osteoarthritis, transient ischemic attack and paroxysmal atrial fibrillation, for which he was prescribed warfarin. He woke on the morning of his presentation with pain and swelling in the elbow, which worsened over 6 hours before he arrived in the emergency department. He had no history of trauma and was non-ambulatory due to chronic pain from arthritis; he required assistance with all transfers from bed to a chair. There was no fever, rash, or other acute arthralgia. He reported that his most recent international normalized ratio (INR) measured 3 days ago was in the therapeutic range.

On physical examination, his temperature was $36.6^{\circ} \mathrm{C}$, pulse

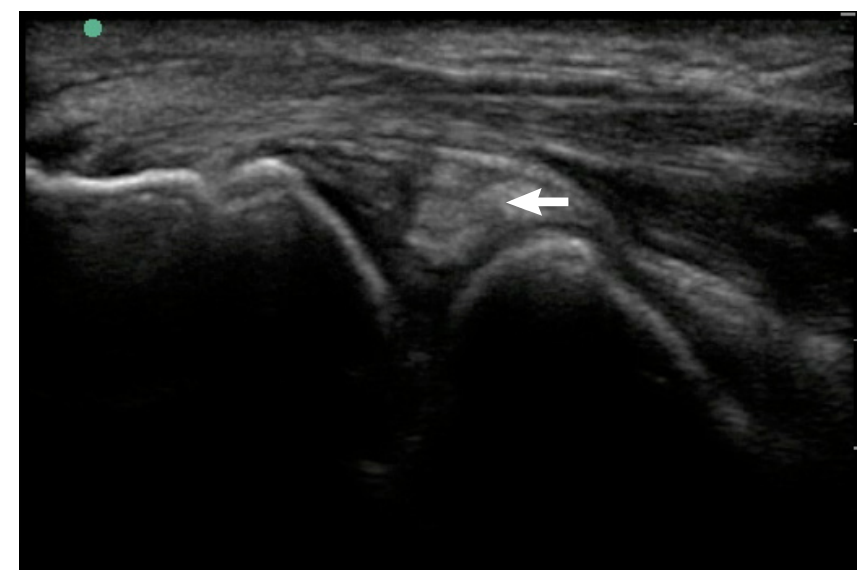

Fig. 1. Longitudinal view of the left elbow demonstrating a heterogeneous, hyperechoic effusion (arrow).
77 beats per minute, respiratory rate 16 per minute, and blood pressure of $157 / 87 \mathrm{mmHg}$. His left elbow was swollen and warm posterolaterally. There was no erythema or ecchymosis. He had no bony tenderness along the joint, upper arm, or forearm. Range of motion of the elbow was markedly limited secondary to pain.

The treating physician performed point-of-care ultrasound to evaluate the swelling and suspected effusion. Using a high-frequency 10-5 MHz transducer (Sonosite, Bothell, WA, USA), both lateral longitudinal and posterior transverse images of the joint were obtained. The ultrasound images demonstrated a complex, compressible effusion with mixed hyperechoic and hypoechoic content, consistent with a hemarthrosis (Figs. 1, 2). ${ }^{2-4}$ No layering fluid was appreciated to suggest lipohemarthrosis from an intraarticular fracture. Radiographs of the elbow were subsequently

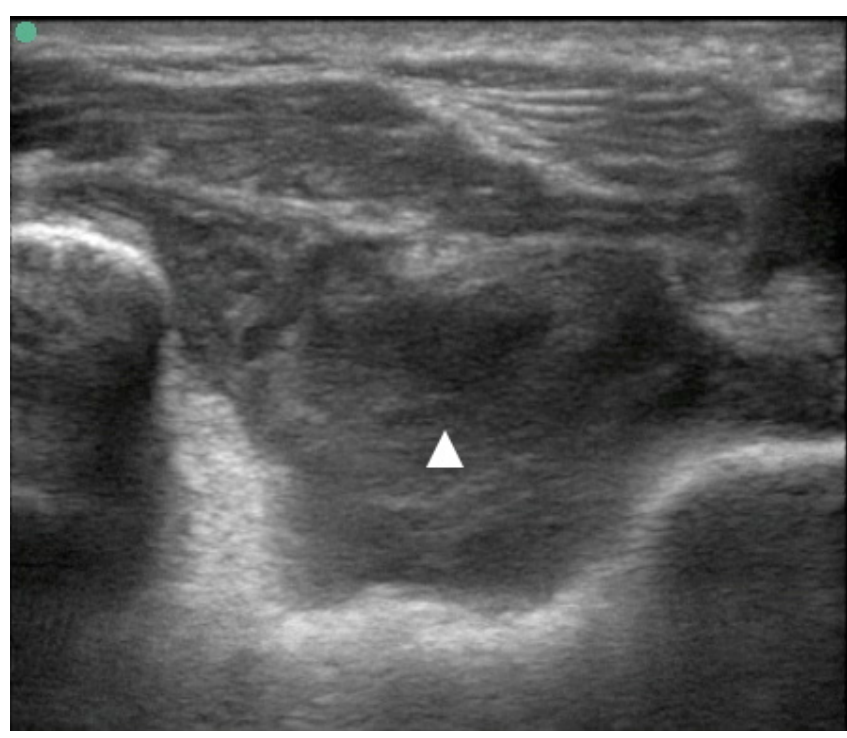

Fig. 2. Posterior transverse view of the elbow with hemarthrosis (triangle).

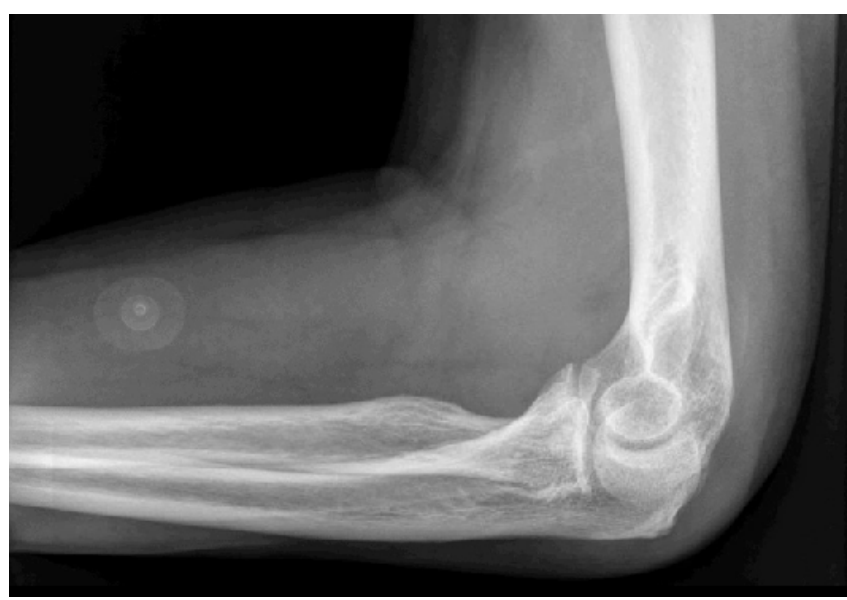

Fig. 3. Lateral X-ray of the elbow demonstrating soft tissue swelling and effusion without evidence of fracture. 
obtained, which re-demonstrated an effusion without evidence of fracture (Fig. 3). Laboratory studies were subsequently available, and notable for an INR of 7.9 and hemoglobin $12.8 \mathrm{~g} / \mathrm{dL}$.

Given the clinical history, absence of trauma or fever, and markedly supratherapeutic INR, a diagnosis of a spontaneous hemarthrosis was made. Although the elbow is an unusual location for a spontaneous hemarthrosis, the point-of-care ultrasound findings consistent with acute bleeding supported the diagnosis and decision to avoid arthrocentesis to further investigate the cause of the effusion. In the absence of bony tenderness or a history of trauma, the ultrasound findings of bleeding without cortical disruption helped obviate the need for computed tomography or magnetic resonance imaging to evaluate for occult fracture.

The patient was managed conservatively, with a focus on symptom control. He was given oral and intravenous analgesia. The affected extremity was placed in a splint and sling for comfort, with a compression bandage applied to the elbow to facilitate hemostasis. Given the degree of pain associated with the hemarthrosis, his coagulopathy was reversed with oral vitamin $\mathrm{K}$ to accelerate INR normalization. He was discharged with instructions to follow up with his physician in 2 days. In serial follow-up visits, he reported progressive decrease in pain and swelling and ultimately symptom resolution. He and his primary care physician ultimately elected to discontinue anticoagulation.

\section{DISCUSSION}

Hemarthrosis is typically the result of injury or bleeding disorder; other causes include osteoarthritis, septic arthritis, tumor, scurvy, and Charcot joint. ${ }^{2,8}$ Spontaneous hemarthrosis may complicate anticoagulant therapy, but typically affects the knee or ankle. To our knowledge, there are no prior reports of a spontaneous elbow hemarthrosis secondary to a supratherapeutic INR. Elbow hemarthrosis is more common in hemophilia, and can lead to arthropathy and chronic pain.

Point-of-care ultrasound is well-suited to evaluate acute joint pain or injury, and can detect effusion, fracture, and tendon or ligamentous rupture. Elbow ultrasound is performed using a highfrequency transducer. Images in 2 planes are obtained on anterior, lateral, medial, and posterior joint surfaces, with particularly attention to the site of maximal pain or swelling. The elbow is extended to obtain images of the joint anteriorly, and flexed to obtain other views. ${ }^{9}$ Comparison with the unaffected extremity may help to identify abnormalities. A recent report described excellent test performance characteristics of point-of-care ultrasound for the detection of traumatic elbow effusions in the emergency department. ${ }^{10}$ Using ultrasound to evaluate musculoskeletal and joint pain in patients with underlying bleeding disorders may have particular value. In hemophiliacs, clinical examination of a painful joint is unreliable, which may prompt clinicians to perform unnecessary invasive procedures or provide costly empiric factor replacement. ${ }^{9-11}$ Recent studies have demonstrated the limitations of clinical examination for the detection of joint effusion in hemophiliacs with suspected hemarthrosis, with improved diagnostic accuracy using point-of-care ultrasound. ${ }^{8,11}$ Analogously, anticoagulated patients with joint pain may be best evaluated with ultrasound to accurately confirm the presence of effusion, and better characterize its content, as in this case.

In patients taking anticoagulants with a suspected spontaneous hemarthrosis, ultrasound findings supportive of bleeding may afford the clinician additional confidence to defer joint aspiration for diagnostic confirmation or exclusion of other etiologies. UItrasound findings consistent with hemathrosis include the presence of a complex effusion, which can be compressed and displaced with the transducer. Hyperacute bleeding may demonstrate anechoic, simple fluid or a uniformly echogenic appearance." Within minutes to hours, a hemarthrosis may layer out as a superficial hypoechoic layer of plasma with a dependent layer of erythrocytes. Intra-articular fractures may demonstrate a 3-phase effusion on ultrasound, with a superficial layer of echogenic lipid, a central hypoechoic layer of plasma, and dependent red cells.

On the basis of the patient's clinical and imaging findings, a confirmatory arthrocentesis was not performed, but the patient's improvement with warfarin reversal and conservative measures supports the diagnosis of a spontaneous hemarthrosis. Arthrocentesis is typically considered safe in patients with a therapeutic INR, but there is little data to support the safety of aspiration in patients with INR $>5 .^{7,12}$

We report a previously undescribed case of a warfarin-related spontaneous elbow hemarthrosis identified by point-of-care ultrasound in the emergency department. Spontaneous hemarthrosis in the elbow is uncommon. Recognition of hemarthrosis with point-of-care ultrasound obviated the need for arthrocentesis to investigate alternative etiologies of an elbow effusion. Physical examination for effusion in patients with high pre-test probability for hemarthrosis is unreliable. Emergency physicians should use musculoskeletal ultrasound to accurately diagnose and better characterize joint effusions in patients with coagulopathies.

\section{CONFLICT OF INTEREST}

No potential conflict of interest relevant to this article was reported. 


\section{REFERENCES}

1. Nawar EW, Niska RW, Xu J. National Hospital Ambulatory Medical Care Survey: 2005 emergency department summary. Adv Data 2007;(386):1-32.

2. Ceponis A, Wong-Sefidan I, Glass CS, von Drygalski A. Rapid musculoskeletal ultrasound for painful episodes in adult haemophilia patients. Haemophilia 2013;19:790-8.

3. Hauzeur JP, Mathy L, De Maertelaer V. Comparison between clinical evaluation and ultrasonography in detecting hydrarthrosis of the knee. J Rheumatol 1999;26:2681-3.

4. Valley VT, Stahmer SA. Targeted musculoarticular sonography in the detection of joint effusions. Acad Emerg Med 2001;8: 361-7.

5. Dubrovsky AS, Kempinska A, Bank I, Mok E. Accuracy of ultrasonography for determining successful realignment of pediatric forearm fractures. Ann Emerg Med 2015;65:260-5.

6. Adhikari S, Blaivas M. Utility of bedside sonography to distinguish soft tissue abnormalities from joint effusions in the emergency department. J Ultrasound Med 2010;29:519-26.
7. Ahmed I, Gertner E. Safety of arthrocentesis and joint injection in patients receiving anticoagulation at therapeutic levels. Am J Med 2012;125:265-9.

8. Kidder W, Nguyen S, Larios J, Bergstrom J, Ceponis A, von Drygalski A. Point-of-care musculoskeletal ultrasound is critical for the diagnosis of hemarthroses, inflammation and soft tissue abnormalities in adult patients with painful haemophilic arthropathy. Haemophilia 2015;21:530-7.

9. Tagliafico AS, Bignotti B, Martinoli C. Elbow US: anatomy, variants, and scanning technique. Radiology 2015;275:636-50.

10. Rabiner JE, Khine H, Avner JR, Friedman LM, Tsung JW. Accuracy of point-of-care ultrasonography for diagnosis of elbow fractures in children. Ann Emerg Med 2013;61:9-17.

11. Zukotynski K, Jarrin J, Babyn PS, et al. Sonography for assessment of haemophilic arthropathy in children: a systematic protocol. Haemophilia 2007;13:293-304.

12. Thumboo J, O'Duffy JD. A prospective study of the safety of joint and soft tissue aspirations and injections in patients taking warfarin sodium. Arthritis Rheum 1998;41:736-9. 